\title{
MotifAdjuster: a tool for computational reassessment of transcription factor binding site annotations Jens Keilwagen*, Jan Baumbach ${ }^{\dagger}$, Thomas A Kohl ${ }^{\ddagger \S}$ and Ivo Grosse*đ
}

\begin{abstract}
Addresses: *Leibniz Institute of Plant Genetics and Crop Plant Research Gatersleben (IPK), Corrensstraße 3, o6466 Gatersleben, Germany. ${ }^{\dagger}$ International Computer Science Institute, 1947 Center Street, Berkeley, California 94704, USA. *International NRW Graduate School in Bioinformatics and Genome Research, Center for Biotechnology (CeBiTec), Bielefeld University, Universitätsstraße 27, 33615 Bielefeld, Germany. §Institute for Genome Research and Systems Biology (IGS), Center for Biotechnology (CeBiTec), Bielefeld University, Universitätsstraße 27, 33615 Bielefeld, Germany. "Institute of Computer Science, Martin Luther University Halle-Wittenberg, VonSeckendorff-Platz 1, o6120 Halle, Germany.
\end{abstract}

Correspondence: Jens Keilwagen. Email: Jens.Keilwagen@ipk-gatersleben.de

Published: I May 2009

Genome Biology 2009, 10:R46 (doi:I0.I I86/gb-2009-10-5-r46)

The electronic version of this article is the complete one and can be found online at http://genomebiology.com/2009//0/5/R46
Received: 19 February 2009

Revised: 17 April 2009

Accepted: I May 2009

(C) 2009 Keilwagen et al., licensee BioMed Central Ltd.

This is an open access article distributed under the terms of the Creative Commons Attribution License (http://creativecommons.org/licenses/by/2.0), which permits unrestricted use, distribution, and reproduction in any medium, provided the original work is properly cited.

\begin{abstract}
Valuable binding-site annotation data are stored in databases. However, several types of errors can, and do, occur in the process of manually incorporating annotation data from the scientific literature into these databases. Here, we introduce MotifAdjuster http://dig.ipk-gatersleben.de/ MotifAdjuster.html, a tool that helps to detect these errors, and we demonstrate its efficacy on public data sets.
\end{abstract}

\section{Rationale}

The regulation of gene expression involves a complex system of interacting components in all living organisms [1] and is of fundamental interest, for instance, for cell maintenance and development. One level of regulation is realized by DNAbinding transcription factors (TFs). The DNA-binding domain of a TF is capable of recognizing specific binding sites (BSs) in the promoter regions of its target genes [2]. Binding of a TF can induce (activator) or inhibit (repressor) the transcription of its target genes. The general ability to control a target gene may depend on the BS itself, its strand orientation, and its position with respect to the transcription start site. If other BSs are present, the ability of a TF to bind the DNA may additionally depend on strand orientations and positions of these BSs.

One important prerequisite for research on gene regulation is the reliable annotation of BSs. The approximate regions on the double-stranded DNA sequence bound by TFs can be determined by wet-lab experiments such as electrophoretic mobility shift assays (EMSAs) [3], DNAse footprinting [4], enzyme-linked immunosorbent assay (ELISA) [5,6], ChIPchip [7], or mutations of the putative BS and subsequent expression studies. Because TFs bind to double-stranded DNA, the strand annotations of nonpalindromic BSs in the databases are either missing or added, based on manual inspection or predictions from bioinformatics tools such as MEME [8], Gibbs Sampler [9,10], Improbizer [11], SeSiMCMC [12], or A-GLAM [13].

After wet-lab identification, data about transcriptional gene regulatory interactions, including the annotated BSs, are published in the scientific literature. Subsequently, these data are extracted by curation teams and manually entered into databases on transcriptional gene regulation such as CoryneRegNet [14], PRODORIC [15], or RegulonDB [16] for prokaryotes, and AGRIS [17], AthaMap [18], CTCFBSDB [19], JASPAR [20], OregAnno [21], SCPD [22], TRANSFAC [23], 
TRED [24], or TRRD [25] for eukaryotes. Three typical problems may occur during the process of transferring these data.

First, erroneously annotated BS: This error may occur in the original study or during the transfer process from the scientific literature to the databases. A sequence is declared to contain a BS, although, in reality, it does not.

Second, shift of the BS: The BS may be erroneously shifted by one or a few base pairs. This typically happens during the transfer process from the scientific literature to the databases.

Third, missing or wrong strand orientation of the BS: The strand orientation of a BS is often not or incorrectly annotated. For example, all BS orientations are arbitrarily declared to be in $5^{\prime} \rightarrow 3^{\prime}$ direction relative to the target gene in CoryneRegNet and in RegulonDB [14,16].

These problems can strongly affect any of the subsequent analysis steps, such as the inference of sequence motifs from "experimentally verified" data, the calculation of $P$ values for the occurrence of BSs, the detection of putative BSs in genome-wide scans and their experimental validation, or the reconstruction of transcriptional gene-regulatory networks.

Here, we introduce MotifAdjuster, a software tool for detecting potential BS annotation errors and for proposing possible corrections. Existing bioinformatics tools [8-13] are not optimized for this task (Additional data file 1), because they do not allow shifting the BS by using a nonuniform distribution and considering both strands with unequal weights. In contrast, MotifAdjuster allows the user to incorporate prior knowledge about (i) the probability of erroneously annotated BSs, (ii) the distribution of possible shifts, and (iii) the strand preference.

One widely-used model for the representation of BSs is the position weight matrix (PWM) model [8-13,26,27], and many software tools for genome-wide scans of sequence motifs are based on PWM models [26,28,29]. MotifAdjuster is based on a simple mixture model using a PWM model on both strands for the motif sequences and a homogeneous Markov model of order o for the flanking sequences similar to MEME, Gibbs Sampler, Improbizer, SeSiMCMC, or A-GLAM. For a given set of BSs, MotifAdjuster tests whether each sequence contains a BS, and it refines the annotations of position and strand for each BS, if necessary, by maximizing the posterior of the mixture model by using a simple expectation maximization (EM) algorithm.

To test the efficacy of MotifAdjuster, we apply it to seven data sets from CoryneRegNet, and we record for each of them the set of potential annotation errors. For one example, the nitrate regulator NarL, we compare the proposed adjustments with the original literature, with a manual strand rean- notation of the BS strands, and with an independent and hand-curated reannotation provided by PRODORIC. Finally, we test whether the PWM estimated from the adjusted NarL BSs can help to detect unknown BSs in those promoter regions that are known to be bound by NarL, but for which no BS could be predicted in the past.

\section{Algorithm}

In this section, we present the MotifAdjuster algorithm including the mixture model, the prior, and the maximum $a$ posteriori (MAP) estimation of the model parameters given the data.

\section{Mixture model}

We denote a DNA sequence of length $L$ by $\underline{x}:=\left(x_{1}, x_{2}, \ldots, x_{L}\right)$, the nucleotide at position $\ell \in[1, L]$ by $x \ell \in\{A, C, G, T\}$, and the reverse complement of $\underline{x}$ by $\underline{x}^{R C}$. For modeling a BS $\underline{x}$ of length $w$, we use a PWM model, which assumes that the nucleotides at all positions are statistically independent of each other, resulting in an additive log-likelihood

$$
\log P_{f}(\underline{x} \mid \underline{\lambda}):=\sum_{\ell=1}^{w} \log P_{\ell}\left(x_{\ell} \mid \underline{\lambda}\right):=\sum_{\ell=1}^{w} \lambda_{x_{\ell}}^{\ell}
$$

of sequence $\underline{x}$ given the model parameters $\underline{\lambda}[30,31]$, where the subscript $f$ stands for foreground. Here, $\lambda_{a}^{\ell}$ denotes the logarithm of the probability of finding nucleotide $a \in\{A, C, G$, $T\}$ at position $\ell, \underline{\lambda \ell}$ denotes the four-dimensional vector $\left(\lambda_{A}^{\ell}, \ldots, \lambda_{T}^{\ell}\right)^{T}$, and $\underline{\lambda}$ denotes the $(4 \times w)$ matrix, that is, $\underline{\lambda}$ denotes the PWM [32-36].

For modeling the flanking sequences, we use a homogeneous Markov model of order o, which assumes that all nucleotides are statistically independent, resulting in an additive log-likelihood

$$
\log P_{b}(\underline{x} \mid \underline{\tau}):=\sum_{\ell=1}^{L} \log P\left(x_{\ell} \mid \underline{\tau}\right):=\sum_{\ell=1}^{L} \tau_{x_{\ell}}
$$

of sequence $\underline{x}$ given model parameters $\underline{\tau}$ [32-36], where the subscript $b$ stands for background. Here, $\tau_{a}$ denotes the logarithm of the probability of nucleotide $a$, and $\underline{\tau}$ denotes the vector $\left(\tau_{A}, \ldots, \tau_{T}\right)^{T}$.

For the detection of sequences (i) erroneously annotated as containing BSs, (ii) with shifted BSs, or (iii) with missing or wrong strand annotations, we introduce the three random variables $u_{1}, u_{2}$, and $u_{3}$.

The variable $u_{1}$ handles the possibility that a sequence annotated as containing a BS does not contain a BS. $u_{1}=0$ denotes 
the case that the sequence contains no $\mathrm{BS}$, and $u_{1}=1$ denotes the case that the sequence contains exactly one BS. If the sequence contains one BS, it can be located at different positions and on both strands.

The variable $u_{2}$ handles the possibility of shifts of a BS caused by annotation errors. $u_{2}$ models the start position of the BS in the sequence with respect to the annotated start position. This variable can assume the integer values $\{-s,-(s-1), \ldots, s-1$, $s\}$, where $s$ is the maximal shift of the BS upstream or downstream of the annotated position.

The variable $u_{3}$ handles the possibility that a BS can have two orientations in the double-stranded upstream region of the target gene. According to the notation of CoryneRegNet, $u_{3}=$ 0 denotes the forward strand defined as the strand in $5^{\prime} \rightarrow 3^{\prime}$ direction relative to the target gene, and $u_{3}=1$ denotes the reverse complementary strand.

For shortness of notation, we define $\underline{u}:=\left(u_{1}, u_{2}, u_{3}\right)$. Because we do not know the values of $\underline{u}$, these variables are modeled as hidden variables. We assume that $u_{2}$ and $u_{3}$ are conditionally independent of each other given $u_{1}$; that is, we assume that annotation errors of position and strand are conditionally independent given the occurrence of the BS. We define

$$
P_{h}(\underline{u} \mid \underline{\phi}):=P_{h}\left(u_{1} \mid \underline{\phi_{1}}\right) P_{h}\left(u_{2} \mid u_{1}, \underline{\phi}_{2}\right) P_{h}\left(u_{3} \mid u_{1}, \underline{\phi}_{3}\right),
$$

where the subscript $h$ stands for hidden, and where $\underline{\phi}:=\left(\underline{\phi}_{1}, \underline{\phi}_{2}\right.$, $\underline{\phi}_{3}$ ) denotes the vector of parameters of this distribution. MotifAdjuster allows the user to specify the probability $P_{h}$ $\left(u_{1} \mid \underline{\phi}_{1}\right)$ that a sequence contains (or does not contain) a BS and the probability distribution $P_{h}\left(u_{2} \mid u_{1}, \underline{\phi}_{2}\right)$ for the length of the erroneous shift. In addition, MotifAdjuster estimates the logarithm of the probability that the BS is located on the forward $(v=0)$ or the reverse complementary $(v=1)$ strand, $\phi_{3, v}:=\log P_{h}\left(u_{3}=v \mid u_{1}=1\right)$, from the user-provided data as described in subsection Expectation maximization algorithm.

The hidden values of $\underline{u}$ lead to the likelihood

$$
P_{a}(\underline{x} \mid \underline{\lambda}, \underline{\tau}, \underline{\phi}):=\sum_{\underline{u}} P_{c}(\underline{x} \mid \underline{u}, \underline{\lambda}, \underline{\tau}) \cdot P_{h}(\underline{u} \mid \underline{\phi})
$$

of the data $\underline{x}$ given the model parameters $(\underline{\lambda}, \underline{\tau}, \underline{\phi})$, where the sum runs over all possible values of $\underline{u}$. Here, the subscript $a$ stands for accumulated, and the subscript $c$ stands for composite. In the following, we define the likelihood in close analogy to $[8,37]$. If sequence $\underline{x}$ contains no BS, we assume that $\underline{x}$ is generated by a homogeneous Markov model of order $\mathrm{O}$; that is,

$$
P_{c}\left(\underline{x} \mid u_{1}=0, \underline{\lambda}, \underline{\tau}\right):=P_{b}(\underline{x} \mid \underline{\tau})
$$

If the sequence $\underline{x}$ contains a BS, then $u_{2}$ encodes its start position, $u_{3}$ encodes its strand, and we assume that the nucleotides upstream and downstream of the BS are generated by a homogeneous Markov model of order o, yielding

$$
\begin{aligned}
P_{c}\left(\underline{x} \mid u_{1}=1, u_{2}, u_{3}, \underline{\lambda}, \underline{\tau}\right): & P_{b}\left(x_{1}, \ldots, x_{u_{2}+s} \mid \underline{\tau}\right) \\
& \cdot P_{m}\left(x_{u_{2}+s+1}, \ldots, x_{u_{2}+s+w} \mid u_{3}, \underline{\lambda}\right) \\
& \cdot P_{b}\left(x_{u_{2}+s+w+1}, \ldots, x_{L} \mid \underline{\tau}\right)
\end{aligned}
$$

and

$$
P_{m}\left(\underline{x} \mid u_{3}, \underline{\lambda}\right):=\left\{\begin{array}{cc}
P_{f}(\underline{x} \mid \underline{\lambda}) & , \text { if } u_{3}=0 \\
P_{f}\left(\underline{x}^{R C} \mid \underline{\lambda}\right), \text { if } u_{3}=1
\end{array},\right.
$$

where the subscript $m$ stands for motif.

\section{Prior}

As prior of the parameters of the PWM model, we use the "common choice" [34-36] of a product of transformed Dirichlets

$$
P(\underline{\lambda} \mid \underline{\alpha}):=\prod_{\ell=1}^{w} D\left(\underline{\lambda}^{\ell} \mid \underline{\alpha}^{\ell}\right):=\prod_{\ell=1}^{w} \Gamma\left(\alpha_{.}^{\ell}\right) \prod_{\alpha \in\{A, C, G, T\}} \frac{\exp \left(\alpha_{a}^{\ell} \lambda_{a}^{\ell}\right)}{\Gamma\left(\alpha_{a}^{\ell}\right)}
$$

where $\alpha_{a}^{\ell}$ denotes the positive hyperparameter of $\lambda_{a}^{\ell}$, $\alpha^{\ell}:=\sum_{\alpha \in\{A, C, G, T\}} \alpha_{a}^{\ell}$ denotes the equivalent sample size (ESS) at position $\ell$, which we set to be equal at each position, $\underline{\alpha}^{\ell}$ denotes the four-dimensional vector $\left(\alpha_{A}^{\ell}, \ldots, \alpha_{T}^{\ell}\right)$, and $\underline{\alpha}$ denotes the $(4 \times w)$ matrix $\left(\underline{\alpha}_{1}, \ldots, \underline{\alpha}_{w}\right)$.

The choice of this prior is pragmatic rather than biologically motivated. This prior is conjugate to the likelihood, allowing to write the posterior as a product of transformed Dirichlets. As PWM models are special cases of Bayesian networks, the chosen prior can be understood as a special case of the Bayesian Dirichlet (BD) prior [38].

Analogously, for homogeneous Markov models of order o, we choose a transformed Dirichlet $P(\underline{\tau} \mid \underline{\beta}):=\mathrm{D}(\underline{\tau} \mid \underline{\beta})$, where $\beta_{a}$ denotes the positive hyperparameter of $\tau_{a}$.

MotifAdjuster allows the user to specify $P\left(u_{1} \mid \underline{\phi}_{1}\right)$ and $P\left(u_{2} \mid u_{1}\right.$, $\left.\underline{\phi}_{2}\right)$. In principle, MotifAdjuster allows the user to specify any probability distribution $P\left(u_{2} \mid u_{1}, \underline{\phi}_{2}\right)$ for the length of the erro- 
neous shift, allowing also asymmetric or bimodal distributions, if needed. For an easy and user-friendly execution, MotifAdjuster also offers a discrete and symmetrically truncated Gaussian distribution defined by

$$
P\left(u_{2}=z \mid u_{1}=1, \underline{\phi}_{2}\right) \propto \exp \left(-\frac{z^{2}}{2 \cdot \sigma^{2}}\right)
$$

where $z$ is an integer value ranging from $-s$ to $s$. The real-valued parameter $\sigma$ is similar to the standard deviation of a Gaussian distribution and can be specified by the user, and we denote $_{2}:=(s, \sigma)$.

We expect that some sequences are annotated to contain a BS, although they do not contain a BS in reality, but we believe that the fraction of such incorrectly annotated sequences is small. Hence, we choose $P\left(u_{1}=0 \mid \underline{\phi}_{1}\right)=0.2$ for the studies presented in this article; that is, we assume that only $20 \%$ of the sequences annotated to contain a BS do not contain a BS in reality. We further expect that the annotated position of the BS might be shifted accidentally by a few base pairs, so we choose $s=5$ and a discrete and symmetrically truncated Gaussian distribution with $\sigma=1$. This choice results in a conditional probability of approximately $40 \%$ that the BS is not shifted, of approximately $25 \%$ that it is shifted $1 \mathrm{bp}$, and of approximately $5 \%$ that it is shifted by more than $1 \mathrm{bp}$ upstream or downstream of the annotated start position, respectively, given that a BS is present in sequence $\underline{x}$.

As prior of the parameter ${ }_{3}$, we choose a transformed Dirichlet $P\left({ }_{3} \mid \chi\right):=D\left({ }_{3} \mid \chi\right)$ with $\chi=\left(\gamma_{0}, \gamma_{1}\right)$, where $\gamma_{v}$ denotes the positive hyperparameter of $\phi_{3, v}$ with $v \in\{0,1\}$.

Putting all pieces together, we define the prior of the parameters of the mixture model of Equation (4) by:

$$
P\left(\underline{\lambda}, \underline{\tau}, \underline{\phi}_{3} \mid \underline{\alpha}, \underline{\beta}, \underline{\gamma}\right)=\left(\prod_{\ell=1}^{w} D\left(\underline{\lambda}^{\ell} \mid \underline{\alpha}^{\ell}\right)\right) \cdot D(\underline{\tau} \mid \underline{\beta}) \cdot D\left(\underline{\phi}_{3} \mid \underline{\gamma}\right),
$$

stating that we assume $\underline{\lambda}, \underline{\tau}$, and $\underline{\phi}_{3}$ to be statistically independent.

We denote the ESS of the mixture model chosen before inspecting any database by $\varepsilon$, and we set the ESS of the PWM model to $P\left(u_{1}=\left.1\right|_{1}\right) \cdot \varepsilon$, the positive hyperparameters of the strand parameters to $\gamma_{\mathrm{O}}=\gamma_{1}=\frac{P\left(u_{1}=1 \mid \underline{\phi}_{1}\right)}{2} \cdot \varepsilon$, and the ESS of the homogeneous Markov model of order o to $\left(L-P\left(u_{1}=\right.\right.$ $\left.\left.\left.1\right|_{1}\right) \cdot w\right) \cdot \varepsilon$. For the reassessment of BSs presented in this article, we choose an ESS of $\varepsilon=5$, yielding an ESS of 4 for the PWM model, $\gamma_{0}=\gamma_{1}=2$, and an ESS of 57 for the homogene- ous Markov model of order o. This choice yields $\alpha_{a}^{\ell}=1$ for every $a \in\{A, C, G, T\}$ and every $\ell \in[1, w]$, stating that the chosen prior of the PWM model can be understood as a special case of the BDeu prior [39,40], which in turn is a special case of the BD prior.

\section{Expectation maximization algorithm}

The model parameters of the mixture model defined by Equation (4) cannot be estimated analytically, but any numeric optimization algorithm can be used for maximizing the posterior. One popular optimization algorithm for maximizing the likelihood $P(\mathrm{~S} \mid \underline{\lambda}, \underline{\tau}, \underline{\phi})$ is the EM algorithm [41]. The EM algorithm can be easily modified for maximizing the posterior $P(\underline{\lambda}, \underline{\tau}, \mid S, \underline{\alpha}, \underline{\beta}, \chi)$ of the data set $S$ by iteratively maximizing:

$$
\begin{aligned}
Q\left(\underline{\lambda}, \underline{\tau}, \underline{\phi}, \underline{\lambda}^{(t)}, \underline{\tau}^{(t)}, \underline{\phi}^{(t)} \mid \underline{\alpha}, \underline{\beta}, \underline{\gamma}\right): & =\left(\sum_{\underline{x} \in S} \sum_{\underline{u}} w_{\underline{u}}^{(t)}(\underline{x}) \cdot \log \left(P_{c}(\underline{x} \mid \underline{u}, \underline{\lambda}, \underline{\tau}) P_{h}(\underline{u} \mid \underline{\phi})\right)\right) \\
& +\log P\left(\underline{\lambda}, \underline{\tau}, \underline{\phi}_{3} \mid \underline{\alpha}, \underline{\beta}, \underline{\gamma}\right)
\end{aligned}
$$

with

$$
w_{u}^{(t)}(\underline{x}):=\frac{P_{c}\left(\underline{x} \mid \underline{u}^{(t)} \underline{\tau}^{(t)}\right) P_{h}\left(\underline{u} \mid \underline{\phi}^{(t)}\right)}{P_{a}\left(\underline{x} \mid \underline{\lambda}^{(t)}, \underline{\tau}^{(t)}, \underline{\phi}^{(t)}\right)} .
$$

$\mathrm{Q}\left(\underline{\lambda}, \underline{\tau}, \underline{\lambda}^{(t)}, \underline{\tau}^{(t)}, \underline{\phi}^{(t)} \mid \underline{\alpha}, \underline{\beta}, \underline{\chi}\right)$ can be maximized analytically with respect to $\underline{\lambda}, \underline{\tau}$, and $\mathrm{f}_{3}$, yielding the familiar expressions provided in Additional data file 2. The posterior $P(\underline{\lambda}, \underline{\tau}, \mid S, \underline{\alpha}$, $\underline{\beta}, \gamma)$ increases monotonically with each iteration, implying that the modified EM algorithm converges to the global maximum, a local maximum, or a saddle point. We stop the algorithm if the logarithmic increase of the posterior between two subsequent iterations becomes smaller than $10^{-6}$, restart the algorithm 10 times with randomly chosen initial values of $w_{u}^{(0)}(\underline{x})$, and choose the parameters of that start with the highest posterior, similar to [8,37]. If we restrict $P_{h}\left(u_{2} \mid u_{1}, \mathrm{f}_{2}\right)$ to a uniform distribution over all possible start positions, if we set $P_{h}\left(u_{3} \mid u_{1}=1\right)=0.5$, and if we restrict the background model to be strand symmetric, then we obtain the probabilistic model that is the basis of $[8,37]$.

The flexibility allowed by MotifAdjuster is important for its practical applicability. Typically, the user has prior knowledge about (i) the expected motif occurrence and (ii) the shift distribution, but (iii) no or only limited prior knowledge about the distribution of the BS strand orientation. Hence, we allow the user to specify the logarithm of the probability that a sequence contains a $\mathrm{BS} \phi_{1,0}$, a nonuniform distribution to incorporate the prior knowledge of the shift distribution, and 
we estimate the logarithm of the probability that the BS is located on the forward strand $\phi_{3,0}$ from the data. This setting allows MotifAdjuster to work, without additional intervention, also in the two extreme cases that the BSs lie predominantly either on the forward or on the reverse complementary strand.

Because of the open source license of MotifAdjuster, similar mixture models can be derived and implemented easily, for instance, by using other background and motif models such as Markov models of higher order [42-44], Permuted Markov models [45], Bayesian networks [46,47], or their extensions to variable order [48-53].

\section{Case studies}

In this section we present the results of MotifAdjuster applied to seven data sets of Escherichia coli, the validation of MotifAdjuster results for NarL BSs, and the prediction of a novel NarL BS.

\section{Results for seven data sets of Escherichia coli}

For testing the efficacy of MotifAdjuster and improving the annotation of BSs of Escherichia coli, we extract all data sets with at least 30 BSs of length of at most $25 \mathrm{bp}$ from the bacterial gene-regulatory reference database CoryneRegNet 4.o. The choice of at least 30 BSs of length of at most $25 \mathrm{bp}$ is arbitrary, but motivated by the intention that the results of the following study should not be influenced by TFs with an insufficient number of BSs or by TFs with an atypical BS length. Seven data sets of BSs corresponding to the TFs CpxR, Crp, Fis, Fnr, Fur, Lrp, and NarL satisfy these requirements, and we apply MotifAdjuster to each of these seven data sets. We summarize the results obtained by MotifAdjuster in Table 1 , and we provide a complete list of the results in Additional data file 3 .
We find that all of the data sets are considered questionable by MotifAdjuster and, more surprisingly, that $34.5 \%$ of the 536 BS annotations are proposed for removal or shifts. The percentage of questionably annotated BSs ranges from $9.3 \%$ for Fnr to 95.7\% for Fur. MotifAdjuster proposes to remove 51 of the 536 BSs and to shift 134 of the remaining 485 BSs by at least one bp, indicating that, in these seven data sets, erroneous shifts of the annotated BSs are the most frequent annotation error. In particular, the percentage of proposed deletions ranges from 2.2\% (one of 46) for Fur to 27.3\% (nine of 33) for CpxR, and the percentage of proposed shifts ranges from $5.6 \%$ (three of 54) for Fnr to 93.5\% (43 of 46) for Fur. In more detail, we observe a broad range of shift lengths ranging from one shift $4 \mathrm{bp}$ upstream to two shifts $4 \mathrm{bp}$ downstream, with a sharp peak about 0 .

For each of the seven TFs, we analyze whether the adjustments proposed by MotifAdjuster result in an improved motif of the BSs (Figure 1). We compute the sequence logos [54,55] of the original BSs obtained from CoryneRegNet and those of the BSs proposed by MotifAdjuster, which we call original sequence logos and adjusted sequence logos, respectively. Comparing these sequence logos, we find that the adjusted sequence logos show a higher conservation than the original sequence logos in all seven cases. We also compare the sequence logos with consensus sequences obtained from the literature [56-61], and we find that the adjusted sequence logos are more similar to the consensus sequences than the original sequence logos. In addition, we find, for the TFs CpxR, Fur, and NarL, that the adjusted sequence logos allow us to recognize clear motifs that could not be recognized in the original sequence logos obtained from CoryneRegNet.

We investigate whether there exists any systematic dependence of the observed rate of proposed adjustments exists on the number of BSs, the BS length, and the GC content of the

Table I

\begin{tabular}{|c|c|c|c|c|c|c|}
\hline Gene ID & Gene name & No. BS & BS length & No. removed BSs & No. shifted BSs & Percentage \\
\hline b3357 & $c r p$ & 218 & 22 & 20 & 31 & $23.4 \%$ \\
\hline$b / 22 I$ & narL & 74 & 7 & 2 & 11 & $17.6 \%$ \\
\hline b326I & fis & 68 & 21 & 13 & 17 & $44.1 \%$ \\
\hline b/334 & $f n r$ & 54 & 14 & 2 & 3 & $9.3 \%$ \\
\hline b0683 & fur & 46 & 15 & I & 43 & $95.7 \%$ \\
\hline b0889 & $\operatorname{lrp}$ & 43 & 12 & 4 & 23 & $62.8 \%$ \\
\hline b39/2 & $c p \times R$ & 33 & 15 & 9 & 6 & $45.5 \%$ \\
\hline Total & & 536 & & 51 & 134 & $34.5 \%$ \\
\hline
\end{tabular}

Summary of the results of the application of MotifAdjuster to all data sets of CoryneRegNet 4.0 from Escherichia coli with at least 30 BSs and of at most 25 bp length. Columns I and 2 show the gene ID and gene name of the TF; columns 3 and 4 show the number of BSs stored in the database and their lengths; columns 5 and 6 show the number of BSs proposed to be removed and to be shifted; and column 7 shows the percentage of BSs to be removed or shifted. Interestingly, the percentage of proposed adjustments varies strongly from TF to TF, ranging from $9.3 \%$ for Fnr to $95.7 \%$ for Fur. In summary, we find in the complete data set of 536 BSs that 5 I BSs are proposed to be removed and I34 BSs are proposed to be shifted, resulting in $34.5 \%$ of the data set being proposed for adjustments. 


\begin{tabular}{|c|c|c|c|c|c|}
\hline & CpxR & \multicolumn{2}{|l|}{ Crp } & \multicolumn{2}{|c|}{ Fis } \\
\hline $\begin{array}{l}\text { Original } \\
\text { sequence } \\
\text { logo }\end{array}$ & 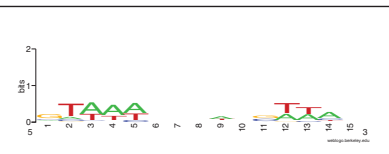 & \multicolumn{2}{|c|}{$\int_{5}^{1}$} & \multicolumn{2}{|c|}{ 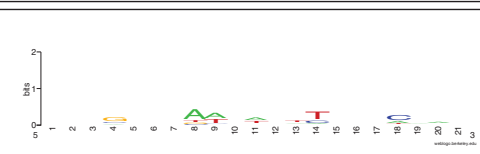 } \\
\hline $\begin{array}{l}\text { Consensus } \\
\text { sequence }\end{array}$ & GTAAANNNNNGTAAA & \multicolumn{2}{|c|}{ TGTGANNNNNNTCACA } & \multicolumn{2}{|c|}{ GNNYWNNWNNYRNNC } \\
\hline \multirow[t]{2}{*}{$\begin{array}{l}\text { Adjusted } \\
\text { sequence } \\
\text { logo }\end{array}$} &  & ${ }^{2}$ & 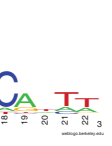 & 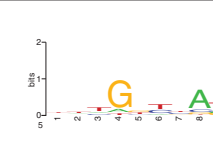 & 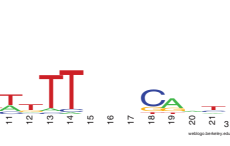 \\
\hline & Fnr & Fur & & Lrp & NarL \\
\hline $\begin{array}{l}\text { Original } \\
\text { sequence } \\
\text { logo }\end{array}$ & ${ }^{2} T T T_{A} A_{1}$ &  & & $\therefore=\sqrt{3}$ &  \\
\hline $\begin{array}{l}\text { Consensus } \\
\text { sequence }\end{array}$ & TTGATNNNNATCAA & GATAATGATAATCATTATC & YAGHA & ATTWTDCTR & TACYYMT \\
\hline $\begin{array}{l}\text { Adjusted } \\
\text { sequence } \\
\text { logo }\end{array}$ & 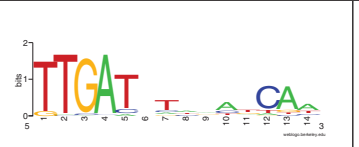 & A AT GATAAT ATT & & & ]$\left._{5}^{2}\right]_{\infty}^{2} A_{\infty}$ \\
\hline
\end{tabular}

Figure I

Comparison of binding-site conservation, showing the original sequence logos, the consensus sequences for the TFs obtained from the literature [56-6I], and the adjusted sequence logos for the data sets of the TFs CpxR, Crp, Fis, Fnr, Fur, Lrp, and NarL. We find in all seven cases that (i) the adjusted sequence logos show a higher conservation than the original sequence logos, (ii) the adjusted sequence logos are more similar to the consensus sequences than to the original sequence logos; and (iii) clear motifs can be recognized in the adjusted sequence logos of the TFs CpxR, Fur, and NarL that could not be recognized in the original sequence logos.

BSs. We find no obvious dependence of the error rate on the number of BSs and on the BS length. Comparing the GC content of the BSs, we find that the GC content of the BSs of all but one TF ranges from $30 \%$ to $40 \%$. However, the GC content of the Fur BSs is only 20\%. This low GC content might be the reason for the unexpectedly high percentage of shifts in this data set, because it is more likely to shift a BS accidentally in a sequence composed of a virtually binary alphabet.

\section{Validation of MotifAdjuster results for NarL}

To evaluate the previous results, we choose NarL as example and scrutinize the proposed reannotations of MotifAdjuster for this case. The nitrate regulator NarL of Escherichia coli is one of the key factors controlling the upregulation of the nitrate respiratory pathway and the downregulation of other respiratory chains. In the absence of oxygen, the energetically most efficient anaerobic respiratory chain uses nitrate and nitrite as electron acceptors [62]. Detection of and adaptation to extracellular nitrate levels are accomplished by complex interactions of a double two-component regulatory system, which consists of the homologous sensory proteins NarQ and NarX, and the homologous TFs NarL and NarP. Depending on the BS arrangement and localization relative to the transcription start site, NarL and NarP act as activators or repres- sors, thereby enabling a flexible control of the expression of nearly 100 genes.

CoryneRegNet stores 74 NarL BSs, each of length 7 bp (Table 1). Of these 74 BSs, only 36 are considered accurate by MotifAdjuster, whereas 38 are considered to be questionable. In 25 cases, MotifAdjuster proposes to switch the strand orientation of the BS; in five cases, it proposes to shift the location of the BS, and for six BSs, it proposes both a switch of strand orientation and a shift of position. In addition, two BSs are proposed for removal. We present a summary of these results in Table 2, we provide a complete list of the results in Additional data file 4 , and we summarize in Table 3 those 13 BSs of the regulator NarL where MotifAdjuster proposes to shift the location of the BS or to remove it from the databases.

To evaluate the accuracy of MotifAdjuster, we check the original literature [63,37] for each of the 13 questionable BS candidates. Comparing both, we find that the proposed annotations agree with those in the literature in all cases but one (BS of gene b1224). That is, in 12 of 13 cases signaled by MotifAdjuster as being questionable, the detected error was indeed caused by an inaccurate transfer from the original literature into the gene-regulatory databases RegulonDB and 
Table 2

\begin{tabular}{l}
$\begin{array}{l}\text { NarL annotation results: } \\
\text { switches }\end{array}$ \\
\begin{tabular}{lcc} 
& No strand switch & Strand switch \\
\hline No position shift & 36 & 25 \\
$\begin{array}{l}\text { Position shift } \\
\text { Removed }\end{array}$ & 5 & 6 \\
\end{tabular} \\
\hline
\end{tabular}

Application of MotifAdjuster to the set of $74 \mathrm{NarL}$ BSs results in adjustments proposed for 38 of these BSs. Two BSs are proposed to be removed from the data set. Of the remaining $36 \mathrm{BSs}, 25 \mathrm{BSs}$ are labeled with a wrong strand annotation but a correct position, and five BSs are proposed to have a correct strand annotation but a wrong position. For six BSs, both strand annotation and position are proposed to be wrong.

CoryneRegNet. Of those 12 questionable BSs, 10 BSs are correctly proposed to be shifted, and two are correctly proposed to be removed.

Turning to the BS of the gene b1224, we find it is published as given in the databases [64], in contrast to the proposal of MotifAdjuster. However, Darwin et al. [67] report that a mutation of this BS has little or no effect on the expression of b1224. Hence, the proposal could possibly be correct, and the BS could be shifted or even be deleted.

In addition, MotifAdjuster checks the strand annotation of BSs and proposes strand switches if needed. To validate these annotations, we cannot use the annotations from RegulonDB and CoryneRegNet, because these databases contain all BSs in $5^{\prime} \rightarrow 3^{\prime}$ direction relative to the target gene. Hence, we consult annotation experts at the Center for Biotechnology in Bielefeld to reannotate the strand orientation of the BSs manually, and we compare the results with those of MotifAdjuster. Interestingly, we find that the strand orientations proposed by MotifAdjuster are in perfect (100\%) agreement with the manually-curated strand orientations. As an independent test of the efficacy of MotifAdjuster for NarL BSs, we use the manually annotated BSs provided by the PRODORIC database [68]. Remarkably, we find also in this case that the results of MotifAdjuster perfectly agree with the annotations.

Another hint that the proposed adjustments of MotifAdjuster could be reasonable is based on the observation that NarL and NarP homodimers bind to a 7-2-7' BS arrangement [61], an inverted repeat structure consisting of a BS on the forward strand, a 2-bp spacer, and a BS on the reverse complementary strand. NarP exclusively binds as homodimer to this 7-2-7' structure. NarL homodimers bind at 7-2-7' sites with highaffinity, but NarL monomers can also bind to a variety of other heptamer arrangements. Instances of this 7-2-7' structure have been reported for four genes: $f d n G, n a p F, \operatorname{nir} B$, and $n r f A[61,65]$. In contrast to this observation, all BSs in CoryneRegNet as well as RegulonDB are annotated to be on the forward strand, including the second half of the inverted repeat. When applied to these four genes, MotifAdjuster proposes all heptamers of the second half of the 7-2-7' structure to be switched to the reverse strand, in agreement with $[61,65]$. In addition, MotifAdjuster proposes six additional 7-

Table 3

NarL binding sites with questionable annotations

\begin{tabular}{|c|c|c|c|c|c|c|c|}
\hline Gene ID & Gene name & BS & Lit. & Occ. & Shift & Strand & Adj. BS \\
\hline b0904 & focA & AATAAAT & [63] & 1 & +1 & Reverse & TATTTAT \\
\hline b0904 & focA & ATAATGC & [63] & I & +1 & Forward & TAATGCT \\
\hline b0904 & focA & ATATCAA & {$[63]$} & I & +1 & Forward & TATCAAT \\
\hline b0904 & focA & CAACTCA & {$[63]$} & 1 & +1 & Forward & AACTCAT \\
\hline b0904 & focA & CATTAAT & [63] & 1 & +1 & Reverse & TATTAAT \\
\hline b0904 & focA & GATCGAT & [63] & 1 & +1 & Reverse & TATCGAT \\
\hline b0904 & focA & GTAATTA & [63] & I & +1 & Forward & TAATTAT \\
\hline b0904 & focA & TATCGGT & {$[63]$} & I & +1 & Reverse & TACCGAT \\
\hline b0904 & focA & TTACTCC & {$[63]$} & I & +1 & Forward & TACTCCG \\
\hline b/ 223 & nark & CACTGTA & [64] & 0 & - & - & - \\
\hline b/ 224 & narG & TAGGAAT & [64] & I & +1 & Reverse & AATTCCT \\
\hline b4070 & $n r f A$ & TGTGGTT & [65] & I & +1 & Reverse & TAACCAC \\
\hline$b 4 / 23$ & $d c u B$ & ATGTTAT & {$[66]$} & 0 & - & - & - \\
\hline
\end{tabular}

Annotated NarL BSs for which MotifAdjuster proposes either to shift the BS or to remove it from the data set. Columns I to 3 contain gene ID, gene name, and the BS (as stored in the database). Column 4 indicates the original literature related to this BS. The following three columns ( 5 through 7) comprise the three possible adjustments suggested by MotifAdjuster, removal, shift, and strand orientation (relative to the target gene). In column 5 , a value of 0 indicates that the BS is proposed for removal, and in column 6 , a positive (negative) value denotes a shift of the BS to the right (left). Finally, column 8 provides the adjusted BS. Interestingly, we find that the two BSs that are proposed to be removed are not mentioned in the original literature, and in 10 of the II cases, the shifted BS is consistent with the BS published in the original literature. In addition, MotifAdjuster also proposes to switch the BS strand in six of the II cases. 
(a) New NarL BS in torC promoter

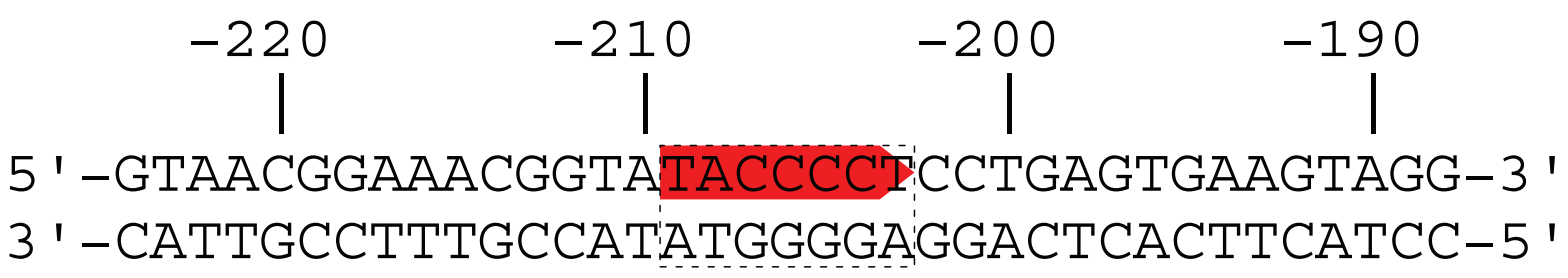

(b) Histogram of all NarL BS positions relative to the start codon

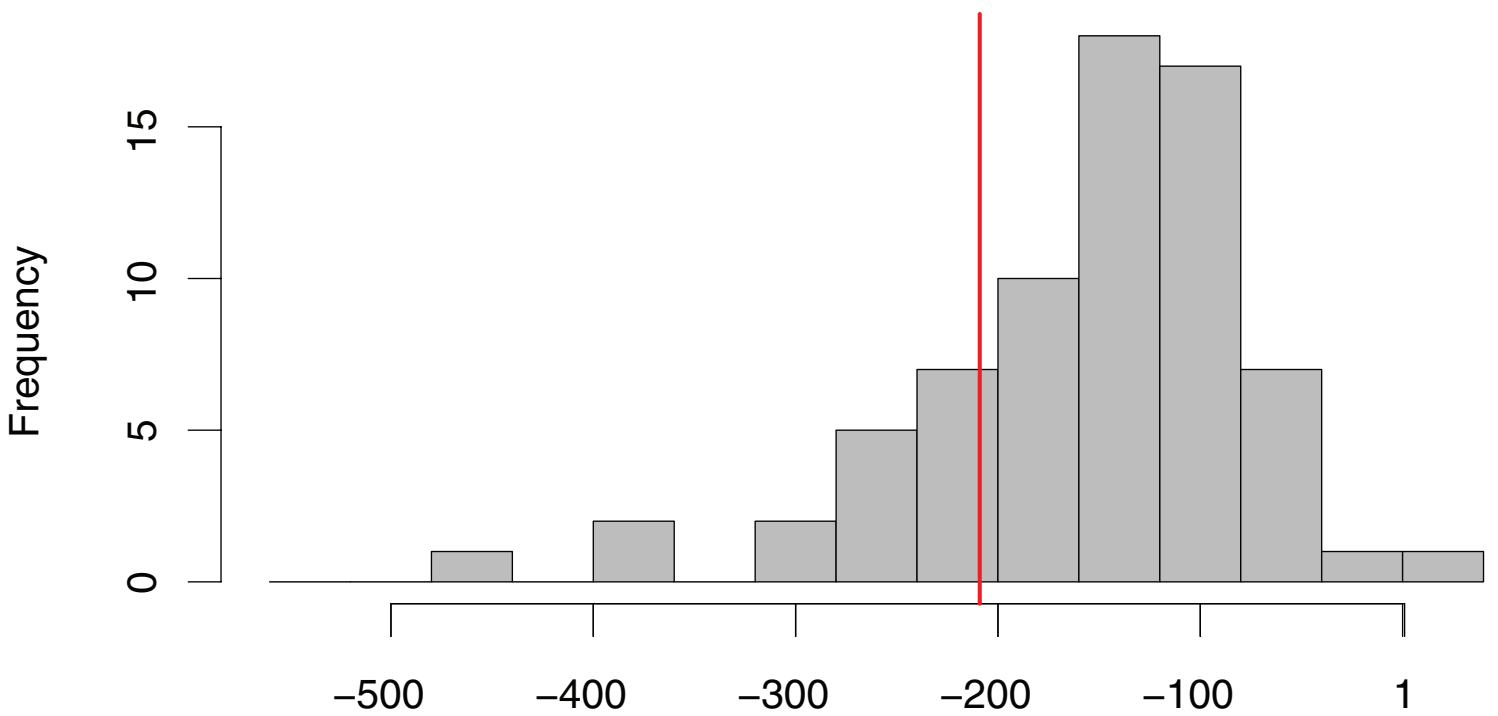

Position relative to start codon

Figure 2

Position of the predicted NarL binding site in the upstream region of torC. The NarL BS TACCCT is located on the forward strand with respect to the target operon torCAD starting at position -209 bp (red color). All positions are relative to the first nucleotide of the start codon of torC. (a) The fragment of the upstream region of the torCAD operon containing the NarL BS predicted by the PWM model trained on the adjusted data set. (b) Histogram of all positions of NarL BSs in the database. The red line indicates the position of the predicted BS.

2-7' BS arrangements, located in the upstream regions of the genes adhE, $\operatorname{asp} A, d c u S, \operatorname{frd} A, h c p$, and norV. The positions and the orientations are presented in Additional data file 4.

\section{Prediction of a novel NarL binding site}

After investigating to which degree MotifAdjuster is capable of finding errors in existing gene-regulatory databases, it is interesting to test whether MotifAdjuster could be helpful for finding novel BSs. The flexibility of BS arrangements and the low motif conservation complicate the computational and manual prediction of NarL BSs by curation teams. This results in several cases in which promoter regions are experimentally verified to be bound by NarL, but in which no NarL BS could be detected [69,70]. Examples of such genes are
caiF [71], torC [72], nikA [73], ubiC [74], and $f d h F$ [75]. We extract the upstream regions of these genes, where an upstream sequence is defined by CoryneRegNet as the sequence between positions $-560 \mathrm{bp}$ and $+20 \mathrm{bp}$ relative to the first position of the annotated start codon of the first gene of the target operon. In addition, we extract those upstream regions of Escherichia coli that belong to operons not annotated as being regulated by NarL (background data set).

We investigate whether we can now detect NarL BSs based on the adjusted data set that could not be detected based on the original data set from CoryneRegNet. For that purpose, we estimate the parameters $\underline{\lambda}$ of the PWM model on the adjusted data set as proposed by MotifAdjuster and $\tau$ of the homogene- 
ous Markov model on the background data set. From the adjusted PWM, we build a mixture model over both strands with the same probability for each strand; that is, $\exp \left(\phi_{3,0}\right)=$ $\exp \left(\phi_{3,1}\right)=0.5$. For the classification of an unknown heptamer $\underline{x}$, we build a simple likelihood-ratio classifier with these parameters $\underline{\lambda}, \underline{\tau},{ }_{3}$ and define the log-likelihood ratio by

$$
r(\underline{x}):=\log \left(\frac{P_{m}\left(\underline{x} \mid \underline{\lambda}, \underline{\phi}_{3}\right)}{P_{b}(\underline{x} \mid \underline{\tau})}\right) .
$$

For an upstream region, we compute $r_{\max }$ defined as the highest log-likelihood ratio of any heptamer $x$ in this upstream region. We compute the $P$ value of a potential BS $\underline{x}$ with value $r(\underline{x})$ as fraction of the background sequences whose $r_{\max }$-values exceed $r(\underline{x})$.

With this classifier, a significant NarL BS can now be detected in the upstream region of torC. Figure 2a shows the doublestranded DNA fragment with the predicted BS (TACCCCT) located on the forward strand starting at $-209 \mathrm{bp}$ relative to the start codon, and at -181 bp relative to the annotated transcription start site [76]. The distance of the predicted BS to the start codon agrees with the distance distribution of previously known NarL BS (Figure 2b), providing additional evidence for the predicted BS. This finding closes the gap between sequence-analysis and gene-expression studies, as the torCAD operon consists of three genes that are essential for the trimethylamine $N$-oxide (TMAO) respiratory pathway [76]. TMAO is present as an osmoprotector in tissues of invertebrates and can be used as respiratory electron acceptor by Escherichia coli. Transcriptional regulation of this operon by NarL binding to the proposed BS would explain nitratedependent repression of TMAO-terminal reductase (TorA) activity under anaerobic conditions [72], thereby linking TMAO and nitrate respiration.

\section{Conclusions}

Gene-regulatory databases, such as AGRIS, AthaMap, CoryneRegNet, CTCFBSDB, JASPAR, ORegAnno, PRODORIC, RegulonDB, SCPD, TRANSFAC, TRED, or TRRD store valuable information about gene-regulatory networks, including TFs and their BSs. These BSs are usually manually extracted from the original literature and subsequently stored in databases. The whole pipeline of wet-lab BS identification and annotation, publication, and manual transfer from the scientific literature to data repositories is not just time consuming but also error prone, leading to many false annotations currently present in databases.

MotifAdjuster is a software tool that supports the (re-)annotation process of BSs in silico. It can be applied as a qualityassurance tool for monitoring putative errors in existing BS repositories and for assisting with a manual strand annotation. MotifAdjuster maximizes the posterior of the parameters of a simple mixture model by considering the possibilities that (i) a sequence being annotated as containing a BS in reality does not contain a BS; (ii) the annotated BS is erroneously shifted by a few base pairs; and (iii) the annotated BS is erroneously located on the false strand and must be reverse complemented. In contrast to existing de-novo motif-discovery algorithms, MotifAdjuster allows the user to specify the probability of finding a BS in a sequence and to specify a nonuniform shift distribution.

We apply MotifAdjuster to seven data sets of BSs for the TFs CpxR, Crp, Fis, Fnr, Fur, Lrp, and NarL with a total of 536 BSs, and we find 51 BSs proposed for removal and 134 BSs proposed for shifts. In total, this results in $34.5 \%$ of the BSs being proposed for adjustments. We choose NarL as an example to scrutinize the proposed reannotations of MotifAdjuster. Checking the original literature for each of the 13 cases shows that the proposed deletions and shifts of MotifAdjuster are in agreement with the published data. Comparing the strand annotation of MotifAdjuster with independent information indicates that the proposals of MotifAdjuster are in accordance with human expertise. Furthermore, MotifAdjuster enables the detection of a novel BS responsible for the regulation of the tor $C A D$ operon, finally augmenting experimental evidence of its NarL regulation. MotifAdjuster is an open-source software tool that can be downloaded, extended easily if needed, and used for computational reassessments of BS annotations.

\section{Availability and requirements}

Project name: MotifAdjuster, project home page: [77], operating system(s): platform independent. Programming language: Java 1.5. Requirements: Jstacs 1.2.2. License: GNU General Public License version 3.

\section{Abbreviations}

BS: binding site; EM: expectation maximization; ESS: equivalent sample size; MAP: maximum a posteriori; PWM: position weight matrix; TF: transcription factor.

\section{Authors' contributions}

JK and IG developed the basic idea, and JK implemented MotifAdjuster. JB and TK provided the data. All authors contributed to data analysis, writing, and approved the final manuscript.

\section{Additional data files}

The following additional data are available with the online version of this article. Additional data file 1 contains a comparison of de-novo motif-discovery tools including MEME, RecursiveSampler, Improbizer, SeSiMCMC, A-GLAM, and MotifAdjuster for the reannotation of NarL. Additional data file 2 contains a detailed description of the MAP parameter 
estimators of the model. Additional data file 3 contains a list of MotifAdjuster results for all seven data sets. Additional data file 4 contains a list of MotifAdjuster results compared with the original input of CoryneRegNet and RegulonDB for the TF NarL.

\section{Acknowledgements}

We thank Lothar Altschmied, Helmut Bäumlein, Karina Brinkrolf, Linda Götz, Jan Grau, Astrid Junker, Gudrun Mönke, Michaela Mohr, Stefan Posch, Yvonne Pöschl, Sven Rahmann, Michael Seifert, Marc Strickert, and Andreas Tauch for helpful discussions, two anonymous reviewers for their valuable comments, Alexander Goesmann, Achim Neumann, and Ralf Nolte for expert technical support, and Richard Münch for his help with the RegulonDB data. J.B. greatly appreciates the support of the German Academic Exchange Service (DAAD). This work was supported by grant 03 I2706A by the German Ministry of Education and Research (BMBF) and XP3624HP/0606T by the Ministry of Culture of Saxony-Anhalt.

\section{References}

I. Babu MM, Teichmann SA: Evolution of transcription factors and the gene regulatory network in Escherichia coli. Nucleic Acids Res 2003, 31: 1234-1244.

2. Pabo CO, Sauer RT: Transcription factors: structural families and principles of DNA recognition. Annu Rev Biochem 1992, 6I:1053-1095

3. Hellman LM, Fried MG: Electrophoretic mobility shift assay (EMSA) for detecting protein-nucleic acid interactions. Nat Protoc 2007, 2:|849-186|.

4. Galas DJ, Schmitz A: DNAse footprinting: a simple method for the detection of protein-DNA binding specificity. Nucleic Acids Res 1978, 5:3157-3170.

5. Benotmane AM, Hoylaerts MF, Collen D, Belayew A: Nonisotopic quantitative analysis of protein-DNA interactions at equilibrium. Analyt Biochem 1997, 250: 18I-185.

6. Mönke G, Altschmied L, Tewes A, Reidt W, Mock HP, Bäumlein H, Conrad U: Seed-specific transcription factors ABI3 and FUS3: molecular interaction with DNA. Planta 2004, 219:158-166.

7. Sun LV, Chen L, Greil F, Negre N, Li TR, Cavalli G, Zhao H, Steensel BV, White KP: Protein-DNA interaction mapping using genomic tiling path microarrays in Drosophila. Proc Natl Acad Sci USA 2003, 100:9428-9433.

8. Bailey TL, Elkan C: Fitting a mixture model by expectation maximization to discover motifs in biopolymers. Proc Int Conf Intell Syst Mol Biol 1994, 2:28-36.

9. Lawrence CE, Altschul SF, Boguski MS, Liu JS, Neuwald AF, Wootton JC: Detecting subtle sequence signals: a Gibbs sampling strategy for multiple alignment. Science 1993, 262:208-214.

10. Thompson W, Rouchka EC, Lawrence CE: Gibbs Recursive Sampler: finding transcription factor binding sites. Nucleic Acids Res 2003, 3 1:3580-3585.

II. Ao W, Gaudet J, Kent WJ, Muttumu S, Mango SE: Environmentally induced foregut remodeling by PHA-4/FoxA and DAF-I2I NHR. Science 2004, 305: 1743-1746.

12. Favorov AV, Gelfand MS, Gerasimova AV, Ravcheev DA, Mironov AA, Makeev VJ: A Gibbs sampler for identification of symmetrically structured, spaced DNA motifs with improved estimation of the signal length. Bioinformatics 2005, 21 :2240-2245.

13. Kim NK, Tharakaraman K, Marino-Ramirez L, Spouge J: Finding sequence motifs with Bayesian models incorporating positional information: an application to transcription factor binding sites. BMC Bioinformatics 2008, 9:262.

14. Baumbach J, Wittkop T, Kleindt CK, Tauch A: Integrated analysis and reconstruction of microbial transcriptional gene regulatory networks using CoryneRegNet. Nature Protocols 2009 in press.

15. Münch R, Hiller K, Barg H, Heldt D, Linz S, Wingender E, Jahn D: PRODORIC: prokaryotic database of gene regulation. Nucleic Acids Res 2003, 3 1:266-269.

16. Gama-Castro S, Jiménez-Jacinto V, Peralta-Gil M, Santos-Zavaleta A, Peñaloza-Spinola MI, Contreras-Moreira B, Segura-Salazar J, MuñizRascado L, Martínez-Flores I, Salgado H, Bonavides-Martínez C,
Abreu-Goodger C, Rodríguez-Penagos C, Miranda-Ríos J, Morett E, Merino E, Huerta AM, Treviño-Quintanilla L, Collado-Vides J: ReguIonDB (version 6.0): gene regulation model of Escherichia coli $\mathrm{K}-\mathrm{I} 2$ beyond transcription, active (experimental) annotated promoters and Textpresso navigation. Nucleic Acids Res 2008, 36:DI20-DI24.

17. Palaniswamy SK, James S, Sun H, Lamb RS, Davuluri RV, Grotewold E: AGRIS and AtRegNet: a platform to link cis-regulatory elements and transcription factors into regulatory networks. Plant Physiol 2006, 140:818-829.

18. Bülow L, Engelmann S, Schindler M, Hehl R: AthaMap, integrating transcriptional and post-transcriptional data. Nculeic Acids Res 2009, 37:D983-D986.

19. Bao L, Zhou M, Cui Y: CTCFBSDB: a CTCF-binding site database for characterization of vertebrate genomic insulators. Nucleic Acids Res 2008, 36:D83-D87.

20. Sandelin A, Alkema W, Engström P, Wasserman WW, Lenhard B: JASPAR: an open-access database for eukaryotic transcription factor binding profiles. Nucleic Acids Res 2004, 32:D91-D94.

21. Montgomery SB, Griffith OL, Sleumer MC, Bergman CM, Bilenky M, Pleasance ED, Prychyna Y, Zhang X, Jones SJM: ORegAnno: an open access database and curation system for literaturederived promoters, transcription factor binding sites and regulatory variation. Bioinformatics 2006, 22:637-640.

22. Zhu J, Zhang M: SCPD: a promoter database of the yeast Saccharomyces cerevisiae. Bioinformatics |999, |5:607-6II.

23. Matys V, Kel-Margoulis OV, Fricke E, Liebich I, Land S, Barre-Dirrie A, Reuter I, Chekmenev D, Krull M, Hornischer K, Voss N, Stegmaier $P$, Lewicki-Potapov B, Saxel $H$, Kel AE, Wingender E: TRANSFAC and its module TRANSCompel: transcriptional gene regulation in eukaryotes. Nucleic Acids Res 2006, 34:DI08-DI I0.

24. Jiang $C$, Xuan Z, Zhao F, Zhang MQ: TRED: a transcriptional regulatory element database, new entries and other development. Nucleic Acids Res 2007, 35:DI37-DI40.

25. Kolchanov NA, Ignatieva EV, Ananko EA, Podkolodnaya OA, Stepanenko IL, Merkulova TI, Pozdnyakov MA, Podkolodny NL, Naumochkin AN, Romashchenko AG: Transcription Regulatory Regions Database (TRRD): its status in 2002. Nucleic Acids Res 2002, 30:312-317.

26. Kel AE, Gössling E, Reuter I, Cheremushkin E, Kel-Margoulis OV, Wingender E: MATCH: a tool for searching transcription factor binding sites in DNA sequences. Nucleic Acids Res 2003, 3I:3576-3579.

27. Tompa M, Li N, Bailey TL, Church GM, Moor BD, Eskin E, Favorov AV, Frith MC, Fu Y, Kent W], Makeev VJ, Mironov AA, Noble WS, Pavesi G, Pesole G, Régnier M, Simonis N, Sinha S, Thijs G, van Helden J, Vandenbogaert M, Weng Z, Workman C, Ye C, Zhu Z: Assessing computational tools for the discovery of transcription factor binding sites. Nat Biotechnol 2005, 23: I37-I 44.

28. Beckstette M, Homann R, Giegerich R, Kurtz S: Fast index based algorithms and software for matching position specific scoring matrices. BMC Bioinformatics 2006, 7:389.

29. Münch R, Hiller K, Grote A, Scheer M, Klein J, Schobert M, Jahn D: Virtual Footprint and PRODORIC: an integrative framework for regulon prediction in prokaryotes. Bioinformatics 2005, 21:4187-4189.

30. Stormo G, Schneider T, Gold L, Ehrenfeucht A: Use of the "Perceptron" algorithm to distinguish translational initiation sites. Nucleic Acids Res 1982, 10:2997-3010.

31. Staden R: Computer methods to locate signals in nucleic acid sequences. Nucleic Acids Res 1984, I 2:505-5 I9.

32. Bernardo JM, Smith AFM: Bayesian Theory New York: John Wiley \& Sons; 1994

33. Thiesson B: Accelerated quantification of Bayesian networks with incomplete data. In Proceedings of First International Conference on Knowledge Discovery and Data Mining (KDD-95): August 20-2I 1995 Edited by: Fayyad U, Uthurusamy R. Montreal: AAAI Press; 1995:306-31।.

34. MacKay DJ: Choice of basis for Laplace approximation. Machine Learning 1998, 33:77-86.

35. Heckerman D: A Tutorial on Learning with Bayesian Networks. Tech. Rep. MSR-TR-95-06, Microsoft Research 1995.

36. Meila M, Jordan Ml: Learning with mixtures of trees. J Machine Learning Res 2000, I: I-48.

37. Lawrence CE, Reilly AA: An expectation maximization (EM) algorithm for the identification and characterization of common sites in unaligned biopolymer sequences. Proteins Struct Funct Genet 1990, 7:41-5I. 
38. Cooper GF, Herskovits E: A Bayesian method for the induction of probabilistic networks from data. Machine Learning 1992, 9:309-347

39. Buntine WL: Operations for learning with graphical models. J Artific Intelligence Res 1994, 2:159-225.

40. Heckerman D, Geiger D, Chickering D: Learning Bayesian Networks: The Combination of Knowledge and Statistical Data Tech. rep., Microsoft Research, Redmond, WA: Advanced Technology Division; 1995.

4I. Dempster AP, Laird NM, Rubin DB: Maximum likelihood from incomplete data via the EM algorithm. J R Stat Soc Series B 1977, 39:1-22.

42. Zhang $M$, Marr T: A weight array method for splicing signals analysis. Comput Appl Biosci 1993, 9:499-509.

43. Salzberg SL: On comparing classifiers: pitfalls to avoid and a recommended approach. Data Mining Knowledge Discov 1997, I:317-328.

44. Thijs G, Lescot M, Marchal K, Rombauts S, De Moor B, Rouze P, Moreau $Y$ : A higher-order background model improves the detection of promoter regulatory elements by Gibbs sampling. Bioinformatics 200।, | 7: | ||3-|| 22.

45. Ellrott K, Yang C, Sladek FM, Jiang T: Identifying transcription factor binding sites through Markov chain optimization. Bioinformatics 2002, I 8:SI00-SI09.

46. Barash Y, Elidan G, Friedman N, Kaplan T: Modeling dependencies in protein-DNA binding sites. Proceedings of Seventh Annual International Conference on Computational Molecular Biology 2003:28-37.

47. Castelo R, Guigo R: Splice site identification by idIBNs. Bioinformatics 2004, 20:169-76.

48. Rissanen J: A universal data compression system. IEEE Trans Inform Theory 1983, 29:656-664.

49. Ron $D$, Singer $Y$, Tishby $N$ : The power of amnesia: learning probabilistic automata with variable memory length. Machine Learning 1996, 25: I I7-I49.

50. Boutilier C, Friedman N, Goldszmidt M, Koller D: Context-specific Independence in Bayesian networks. Proceedings of the Twelfth Annual Conference on Uncertainty in Artificial Intelligence 1996: I I5-I23.

5I. Bühlmann P: Model Selection for Variable Length Markov Chains and Tuning the Context Algorithm Tech. Rep. 82, Statistics, Zurich: ETH Zentrum; 1997.

52. Zhao $X$, Huang $H$, Speed TP: Finding short DNA motifs using permuted Markov models. J Comput Biol 2005, I 2:894-906.

53. Ben-Gal I, Shani A, Gohr A, Grau J, Arviv S, Shmilovici A, Posch S, Grosse I: Identification of transcription factor binding sites with variable-order Bayesian networks. Bioinformatics 2005, 2l:2657-2666.

54. Schneider TD, Stephens RM: Sequence logos: a new way to display consensus sequences. Nucleic Acids Res 1990, 18:6097-6100.

55. Crooks GE, Hon G, Chandonia JM, Brenner SE: WebLogo: a sequence logo generator. Genome Res 2004, I 4: I I88-I I 90.

56. De Wulf P, McGuire AM, Liu X, Lin ECC: Genome-wide profiling of promoter recognition by the two-component response regulator CpxR-P in Escherichia coli. J Biol Chem 2002, 277:26652-2666I.

57. Körner H, Sofia HJ, Zumft WG: Phylogeny of the bacterial superfamily of Crp-Fnr transcription regulators: exploiting the metabolic spectrum by controlling alternative gene programs. FEMS Microbiol Rev 2003, 27:559-592.

58. Pan CQ, Johnson RC, Sigman DS: Identification of new Fis binding sites by DNA scission with Fis-I, I 0-phenanthroline-copper(I) chimeras. Biochemistry 1996, 35:4326-4333.

59. Baichoo N, Helmann JD: Recognition of DNA by Fur: a reinterpretation of the Fur box consensus sequence. J Bacteriol 2002, I 84:5826-5832.

60. Cui Y, Wang Q, Stormo G, Calvo J: A consensus sequence for binding of Lrp to DNA. J Bacteriol 1995, I 77:4872-4880.

61. Maris AE, Kaczor-Grzeskowiak M, Ma Z, Kopka ML, Gunsalus RP, Dickerson RE: Primary and secondary modes of DNA recognition by the NarL two-component response regulator. Biochemistry 2005, 44:|4538-|4552.

62. Unden G, Bongaerts J: Alternative respiratory pathways of Escherichia coli: energetics and transcriptional regulation in response to electron acceptors. Biochim Biophys Acta 1997, 1320:217-234.

63. Kaiser M, Sawers G: Nitrate repression of the Escherichia coli pfl operon is mediated by the dual sensors NarQ and NarX and the dual regulators NarL and NarP. J Bacteriol 1995, I 77:3647-3655.

64. Li J, Kustu S, Stewart V: In vitro interaction of nitrate-respon- sive regulatory protein NarL with DNA target sequences in the fdnG, narG, nark and frdA operon control regions of Escherichia coli K-I2. J Mol Biol 1994, 24I: I50-I65.

65. Darwin AJ, Tyson KL, Busby SJ, Stewart V: Differential regulation by the homologous response regulators NarL and NarP of Escherichia coli K-I2 depends on DNA binding site arrangement. Mol Microbiol 1997, 25:583-595.

66. Golby P, Kelly DJ, Guest JR, Andrews SC: Transcriptional regulation and organization of the dcuA and dcuB genes, encoding homologous anaerobic C4-dicarboxylate transporters in Escherichia coli. J Bacteriol 1998, I80:6586-6596.

67. Darwin AJ, Li J, Stewart V: Analysis of nitrate regulatory protein NarL-binding sites in the fdnG and narG operon control regions of Escherichia coli K-I2. Mol Microbiol 1996, 20:62 I-632.

68. PRODORIC URL of the Matrix of NarL [http://www.pro doric.de/matrix.php?matrix_acc=MX000003]

69. Overton TW, Griffiths L, Patel MD, Hobman JL, Penn CW, Cole JA Constantinidou $\mathrm{C}$ : Microarray analysis of gene regulation by oxygen, nitrate, nitrite, FNR, NarL and NarP during anaerobic growth of Escherichia coli: new insights into microbial physiology. Biochem Soc Trans 2006, 34: 104-107.

70. Constantinidou C, Hobman JL, Griffiths L, Patel MD, Penn CW, Cole IA, Overton TW: A reassessment of the FNR regulon and transcriptomic analysis of the effects of nitrate, nitrite, NarXL, and NarQP as Escherichia coli KI 2 adapts from aerobic to anaerobic growth. I Biol Chem 2006, 28 I:4802-48I 5.

7I. Eichler K, Buchet A, Lemke R, Kleber HP, Mandrand-Berthelot MA: Identification and characterization of the caiF gene encoding a potential transcriptional activator of carnitine metabolism in Escherichia coli. J Bacteriol 1996, I 78: I248-I 257.

72. luchi S, Lin EC: The narL gene product activates the nitrate reductase operon and represses the fumarate reductase and trimethylamine $\mathbf{N}$-oxide reductase operons in Escherichia coli. Proc Natl Acad Sci USA 1987, 84:390I-3905.

73. Rowe JL, Starnes GL, Chivers PT: Complex transcriptional control links NikABCDE-dependent nickel transport with hydrogenase expression in Escherichia coli. J Bacteriol 2005, 187:6317-6323.

74. Kwon O, Druce-Hoffman M, Meganathan R: Regulation of the ubiquinone (coenzyme $Q$ ) biosynthetic genes ubiCA in Escherichia coli. Curr Microbiol 2005, 50:180-189.

75. Wang H, Gunsalus RP: Coordinate regulation of the Escherichia coli formate dehydrogenase fdnGHI and fdhF genes in response to nitrate, nitrite, and formate: roles for NarL and NarP. I Bacteriol 2003, 1 85:5076-5085

76. Méjean V, lobbi-Nivol C, Lepelletier M, Giordano G, Chippaux M, Pascal MC: TMAO anaerobic respiration in Escherichia coli: involvement of the tor operon. Mol Microbiol 1994 I I:I I69- I I79.

77. Jstacs: A Java Framework for Statistical Analysis and Classification of Biological Sequences [http://www.jstacs.de] 